canonical energy momentum tensor as

$$
\begin{gathered}
m=-\frac{1}{c^{2}} \int T_{44} d V \\
T_{44}=-\frac{i \hbar c}{2}\left(\psi^{\dagger} \gamma_{\frac{1}{2}} \frac{\partial \psi}{\partial x_{4}}-\frac{\partial \psi^{\dagger}}{\partial x_{4}} \gamma_{4} \psi\right) \\
-\sum_{\nu} \frac{\partial A_{\nu}}{\partial x_{4}} \frac{\partial L}{\partial\left(\partial A_{\nu} / \partial x_{4}\right)}+L
\end{gathered}
$$

and would consist, in the case of solutions that are regular everywhere, of finite contributions from both the spinor field and the electromagnetic field. It is clear that different types of solutions should yield different masses corresponding to these solutions. We anticipate, in fact, the appearance of a mass spectrum. ${ }^{1}$

It should also be possible to understand by a theory of this type the reason for the fact that certain kinds of particles, like magnetic poles, do not exist in nature, because it is conceivable that no solutions regular in the interior can be found which would fit to a solution of the exterior corresponding to, say, a magnetic pole.

It appears also reasonable to expect that within the framework of such a theory one may be able to grasp unstable particles, and particles which possess excited states. In fact, the ultimate aim of this effort is to understand all particles, from the electron up to the unstable nuclei of the heaviest elements in terms of excitations of a medium which is described by a variational principle of the type (1).

Work to produce solutions of the field equations $[(3),(4),(5)]$ is under way.

1 Since the basic action principle (1) does not contain any constants from which one can build any constant of the dimension of mass, it is obvious that the anticipated mass spectrum must appear in terms of a smallest mass which remains completely undetermined in the classical theory, and which by similarity transformation of the solutions may be fitted to the experimental value. The situation is analogous to that encountered by $\mathrm{J}$. A Wheeler in his theory of classical geons [Phys. Rev. 97, 511 (1955)], which may be called "particles" built up out of gravitons and photons.

\section{Classification of the Nucleonic States in Deformed Nuclei}

\author{
B. R. Mottelson and S. G. Nilsson
}

CERN, ${ }^{*}$ Theoretical Study Division and the Institutes for Theoretical Physics, Universities of Copenhagen and Lund, Denmark (Received July 7, 1955)

$T$ HE nuclear shell structure and the equilibrium shape of the nucleus are intimately related. Thus, the nuclear distortions are a consequence of the centrifugal pressures exerted by the individual nu- cleons. ${ }^{1}$ In turn the deformation of the nuclear field implies important modifications of the nucleonic motion. ${ }^{2}$ This interplay between nucleonic motion and nuclear distortion is most easily studied in nuclei possessing large equilibrium distortions. In the present note we report a classification of the nucleonic states in the deformed nuclei in the region $150<A<194$. From the ground-state configurations we then calculate the equilibrium deformations..$^{3,4}$

The level spectrum of individual particle motion in an ellipsoidal potential with the inclusion of a spin-orbit force has been calculated as a function of the nuclear eccentricity. ${ }^{5}$ The adjustable parameters in the poten-

TABLE I. The expected ground-state spins, $I_{\text {theo, }}$ obtained from Figs. 1 and 2, are compared with measured nuclear spins taken from reference 6 . The assumed nuclear deformations are deduced

\begin{tabular}{|c|c|c|c|}
\hline Nucleus & $\begin{array}{c}\text { Assumed } \\
\text { deforma- } \\
\text { tions }\end{array}$ & $I_{\text {theo }}$ & $I_{\text {exp }}$ \\
\hline${ }^{88}{ }_{63} \mathrm{Eu}^{151}$ & 0.16 & $3 / 2 \pm, 5 / 2 \pm$ & $5 / 2$ \\
\hline${ }_{63}^{90} \mathrm{Eu}^{153}$ & 0.30 & $5 / 2+, 3 / 2+$ & $5 / 2$ \\
\hline${ }_{94}^{95} \mathrm{~Tb}^{159}$ & 0.31 & $3 / 2+, 5 / 2+$ & $3 / 2$ \\
\hline${ }^{98}{ }_{67} \mathrm{Ho}^{165}$ & 0.30 & $7 / 2-, 1 / 2+$ & $7 / 2$ \\
\hline${ }_{69}^{100} \mathrm{Tm}^{169}$ & 0.28 & $1 / 2+, 7 / 2-$ & $1 / 2$ \\
\hline${ }^{104}{ }_{71} \mathrm{Lu}^{175}$ & 0.28 & $7 / 2+, 5 / 2+$ & $7 / 2$ \\
\hline${ }^{108}{ }_{73} \mathrm{Ta}^{181}$ & 0.23 & $5 / 2+, 7 / 2+$ & $7 / 2$ \\
\hline${ }^{110}{ }_{75} \mathrm{Re}^{185}$ & $0.19^{\mathrm{a}}$ & $9 / 2-,(5 / 2+)$ & $5 / 2$ \\
\hline${ }_{112}{ }_{75} \operatorname{Re}^{187}$ & 0.19 & $9 / 2-,(5 / 2+)$ & $5 / 2$ \\
\hline${ }^{114}{ }_{77} \operatorname{Ir}^{191}$ & 0.14 & $3 / 2+, 1 / 2+, 11 / 2-$ & $3 / 2$ \\
\hline${ }_{116}{ }_{77} \mathrm{Ir}^{193}$ & $0.12^{\mathrm{a}}$ & $3 / 2+, 1 / 2+, 11 / 2-$ & $3 / 2$ \\
\hline${ }^{91}{ }_{64} \mathrm{Gd}^{155}$ & $0.31^{\mathrm{a}}$ & $5 / 2+, 3 / 2-$ & $\geq 3 / 2$ \\
\hline${ }_{64}^{93} \mathrm{Gd}^{157}$ & $0.31^{\mathrm{a}}$ & $3 / 2-, 5 / 2+$ & $\geq 3 / 2$ \\
\hline${ }_{95}^{96} \mathrm{Dy}^{161}$ & $0.31^{\mathrm{a}}$ & $5 / 2-$ & \\
\hline${ }_{97}^{97} \mathrm{Dy}^{163}$ & & $\mathrm{~b}$ & \\
\hline${ }_{68}^{99} \mathrm{Er}^{167}$ & $0.29^{\mathrm{a}}$ & $1 / 2-, 7 / 2+$ & $7 / 2$ \\
\hline${ }^{101}{ }_{70} \mathrm{Yb}^{171}$ & $0.29^{a}$ & $7 / 2+, 1 / 2-$ & $1 / 2$ \\
\hline${ }^{103}{ }_{70} \mathrm{Yb}^{173}$ & $0.29^{\mathrm{a}}$ & $5 / 2-$ & $5 / 2$ \\
\hline${ }^{105}{ }_{72} \mathrm{Hf}^{177}$ & 0.26 & $7 / 2-$ & \\
\hline${ }^{107}{ }_{72} \mathrm{Hf}^{179}$ & 0.27 & $9 / 2+$ & \\
\hline${ }^{109}{ }_{74} \mathrm{~W}^{183}$ & 0.21 & $1 / 2-, 7 / 2-, 3 / 2-$ & $1 / 2$ \\
\hline $111_{76} \mathrm{Os}^{187}$ & $0.18^{a}$ & $1 / 2-, 3 / 2-, 9 / 2+$ & \\
\hline${ }^{113}{ }_{76} \mathrm{Os}^{189}$ & $0.15^{\mathrm{a}}$ & $1 / 2-, 3 / 2-, 11 / 2+$ & $3 / 2$ \\
\hline
\end{tabular}
from the measured intrinsic quadrupole moments where available; in other cases interpolated values are employed.

a Interpolated value.

b Prediction sensitive to assumed deformation.

tial, such as the strength of the spin-orbit force, have been chosen to reproduce as well as possible the observed single-particle spectra in the approximately spherical nuclei near closed shells. ${ }^{6}$ It is found necessary to choose slightly different parameters to reproduce the neutron and proton spectra respectively. For the protons we use the parameters corresponding to Table Ib of reference 5, while for the neutrons the parameters of Table I of the same reference are employed. The differences between these two choices of the parameters are in the general sense of favoring proton orbits of higher angular momentum as compared with the corresponding neutron orbits.

The parts of the spectra relevant to the present discussion are reproduced in Fig. 1, which covers the 
proton orbits from $Z=50$ to $Z=82$, and in Fig. 2, which covers the neutron orbits from $N=82$ to $N=126$. The abscissa is the deformation parameter $\delta$ which is related to the intrinsic electric quadrupole moment $Q_{0}$ by means of the relation

$$
Q_{0}=\frac{4}{5} Z R_{0}^{2} \delta\left(1+\frac{1}{2} \delta+\cdots\right),
$$

where $Z$ is the nuclear charge number, and $R_{0}$ is the mean radius for the nuclear charge distribution, which we take to be $R_{0}=1.2 \times A^{\frac{1}{3}} 10^{-13} \mathrm{~cm}$. The individual orbits are labelled by the parity and the component $\Omega_{p}$ of the nucleon's angular momentum along the nuclear symmetry axis. Each orbit may be occupied twice, corresponding to the two possible signs of $\Omega_{p}$. The absence of degeneracies, except that associated with the sign of $\Omega_{p}$, implies a very simple coupling scheme for the particle motion. Thus, in an odd- $A$

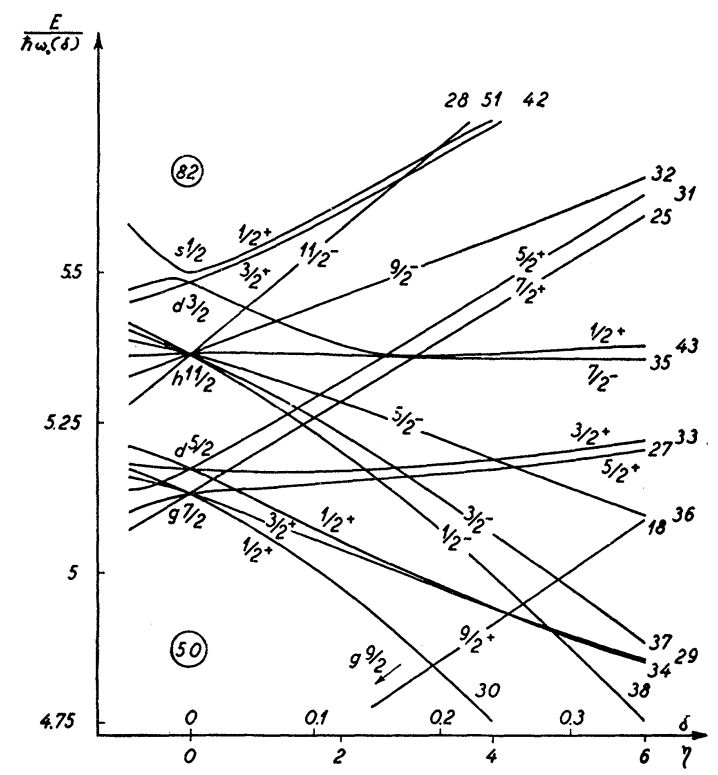

FIG. 1. Spectra for protons from $Z=50$ to $Z=82$ as a function of the nuclear deformation. Only prolate deformations aie shown. The numbers labelling the orbits on the right are arbitrarily assigned and correspond to the notation of reference 5 .

nucleus the ground-state spin is equal to the value of $\Omega_{p}$ for the orbit occupied by the last odd nucleon [except for certain exceptional orbits $\Omega_{p}=\frac{1}{2}$, see (2) and (5)].

It should be noted that the spectra of Figs. 1 and 2 contain many deviations from the usual shell model rules as to the relation of spin and parity in a given shell.

In order to employ these level schemes in the classification of the nuclear states it is necessary to have an estimate of the nuclear equilibrium deformation. We use for this purpose the measured nuclear quadrupole moments $Q_{0}$ and the relation (1). The values of $Q_{0}$ are taken from a recent summary ${ }^{7}$ of Coulomb excitation and lifetime determinations, and the resulting values of $\delta$ are given in Table I.

Employing the observed deformations one can obtain from Figs. 1 and 2 the expected nuclear ground-state spin and parity. The values obtained in this way are compared with the experimental spins in Table I. Where the calculated spectra give several close-lying states, the exact sequence may be somewhat fortuitous, and in such cases several possibilities are listed in column three of Table I. One expects the alternative states to occur as low-lying intrinsic excitations in the nuclear spectra and in many cases these have already been observed. Thus, for example, in $\mathrm{Lu}^{175}$ the $5 / 2+$ state occurs at an excitation of $342 \mathrm{kev} .^{8}$ In $\mathrm{W}^{183}$ the $3 / 2$ - intrinsic excitation occurs at an energy of $209 \mathrm{kev}$, while the $7 / 2$ - state is at $453 \mathrm{kev} .^{9}$

Additional low-lying excited states in the nuclei in the region $A \sim 190$ may result from the nucleonic states

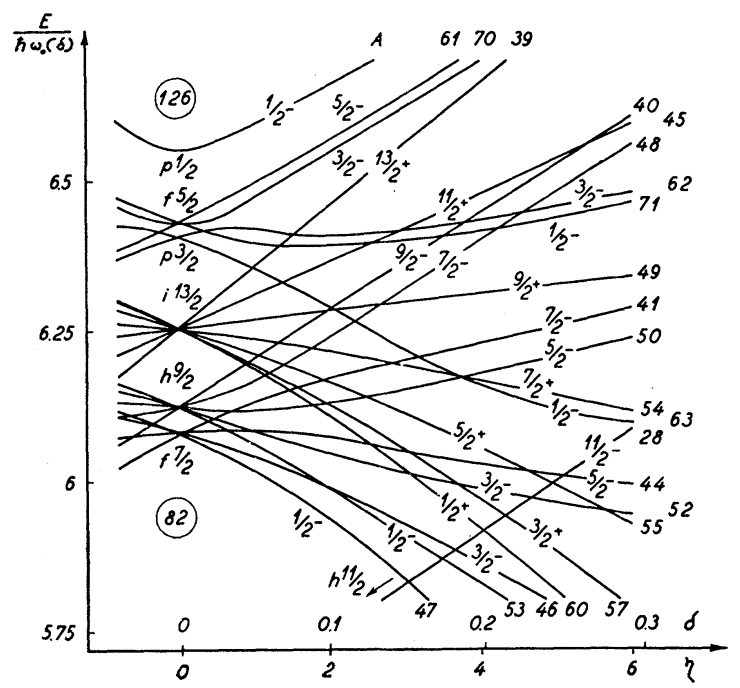

Fig. 2. Same as Fig. 1 but for neutrons from $N=82$ to $N=126$.

of low $\Omega_{p}$ from the next higher shell (these levels are not shown in the figures). Such configurations would be characterized by appreciably larger deformations than in the ground state.

The agreement between the observed spins and those obtained from Figs. 1 and 2 is seen to be excellent and thus to support the coupling scheme employed in the description of the nucleonic motion.

The very great difference in the deformation in $\mathrm{Eu}^{151}$ and $\mathrm{Eu}^{153}$ implies rather different intrinsic spectra in these two isotopes. In $\mathrm{Eu}^{153}$ the ground state has $\Omega_{p}=5 / 2+$, and the excited configuration at $103 \mathrm{kev}$ has the expected $\Omega_{p}=3 / 2+$ character. ${ }^{10}$ Although Eu ${ }^{151}$ also has a ground-state spin $I=5 / 2$, the measured magnetic moment identifies the configuration as $\Omega_{p}$ $=5 / 2-$ (orbit No. 36 in Fig. 1).

The observed magnetic moment and rotational spectrum of $\mathrm{Tm}^{169}$ has been discussed previously ${ }^{11}$ and 
found to be in good agreement with the present classification.

Following the filling of the $7 / 2+$ orbit in $\mathrm{Lu}^{175}$, the next two protons appear to fill the $9 / 2$ - orbit pairwise. The $7 / 2+$ orbit then occurs a second time as a ground state in $\mathrm{Ta}^{181}$, and the $\mathrm{Re}$ isotopes have the $\Omega_{p}=5 / 2+$ ground-state configuration. Although the $9 / 2-$ orbit thus does not occur as a ground-state configuration, its existence is verified by its occurrence as an excited configuration in $\mathrm{Lu}^{175},{ }^{12}$ in $\mathrm{Lu}^{177},{ }^{13}$ and in $\mathrm{Re}^{187} \cdot{ }^{13}$ The occurrence of this small irregularity in the level sequence may be associated with the residual interactions between the nucleons. ${ }^{14}$

The experimental evidence concerning the spins of $\mathrm{Hf}^{177}$ and $\mathrm{Hf}^{179}$ is conflicting. Tentative hyperfine structure measurements ${ }^{15}$ have been interpreted as suggesting a spin of $\frac{1}{2}$ or $\frac{3}{2}$. However, more recent evidence $^{16}$ from the nuclear rotational spectra seems more consistent with the high spins expected from Fig. 2.

In the discussion of the nuclear ground-state spins, we have used the experimentally measured deformation. One may, however, obtain a theoretical estimate of this quantity by considering the total nuclear energy obtained from Figs. 1 and 2 as a function of the deformation and thus obtain the equilibrium shape for each configuration. ${ }^{17}$ It is found that the calculated equilibrium shapes have the observed prolate type of deformations for the nuclei considered in the present note. The calculated deformations for the ground-state configurations are also found to follow rather well the variation of the nuclear distortions as deduced from the observed electric quadrupole moments (see Fig. 3).

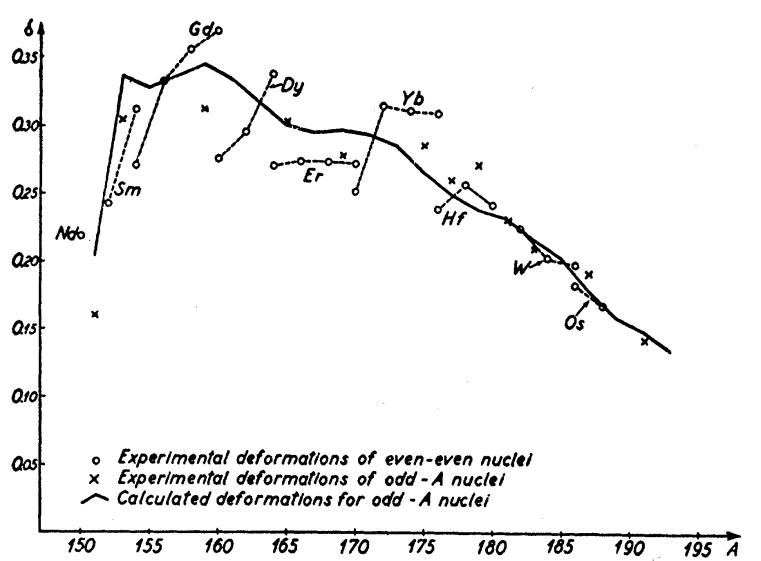

FIG. 3. The calculated ground-state equilibrium deformations are compared with those deduced from the observed intrinsic quadrupole moments.

In particular the dramatic increase in the nuclear deformation which is observed ${ }^{18}$ in going from neutron number $N=88$ to $N=90$ follows as a consequence of the breaking up of the $h_{11 / 2}$ shell which occurs at this point.
During the course of the present work we have enjoyed many illuminating discussions with Dr. A. Bohr, and it is a pleasure to acknowledge the stimulation which he has provided.

* European Organization for Nuclear Research.

1 J. Rainwater, Phys. Rev. 79, 432 (1950).

2 For the detailed formulation of the nuclear model employed, see A. Bohr, Kgl. Danske Videnskab. Selskab. Mat.-fys. Medd. 26, No. 14 (1952); and A. Bohr and B. Mottelson, Kgl. Danske Videnskab. Selskab, Mat.-fys. Medd. 27, No. 16 (1953).

${ }^{3} \mathrm{~A}$ more complete report including a discussion of magnetic moments, excited states, and transition probabilities will be submitted to Kgl. Danske Videnskab. Selskab, Mat.-fys. Medd.

${ }^{4} \mathrm{~A}$ classification of these nucleonic states, which is in many respects similar to that given here, has been independently suggested by K. Gottfried, thesis, Massachusetts Institute of Technology, June, 1955 (unpublished).

${ }^{5}$ S. G. Nilsson, Kgl. Danske Videnskab. Selskab, Mat.-fys. Medd. 29, No. 16 (1955).

${ }^{6}$ M. G. Mayer and J. H. D. Jensen, Elementary Theory of Nuclear Shell Structure (John Wiley and Sons, Inc., New York and London, 1955)

${ }^{7}$ A. Bohr and B. R. Mottelson, Kgl. Danske Videnskab. Selskab, Mat.-fys. Medd. (to be published).

8 Burford, Perkins, and Haynes, Phys. Rev. 95, 303(A) (1954), and private communication.

${ }^{9}$ Murray, Boehm, Marmier, and DuMond, Phys. Rev. 97, 1007 (1955).

${ }_{10}$ M. R. Lee and R. Katz, Phys. Rev. 93, 155 (1954); R. L. Graham and J. Walker Phys. Rev. 94, 794(A) (1954); N. Marty, Compt. rend. 238, 2516 (1954).

${ }^{11}$ B. R. Mottelson and S. G. Nilsson, Z. Physik 141, 217 (1955)

12 N. Marty, Compt. rend. 240, 963 (1955); H. de Waard, Phil. Mag. 46, 445 (1955); Akerlind, Hartmann, and Wiedling, Phil. Mag. 46, 448 (1955).

${ }^{13}$ See, e. g., Hollander, Perlman, and Seaborg, Revs. Modern Phys. 25, 469 (1953).

14 The effect of such residual interactions on the nuclear moments of inertia is discussed in reference 7 .

${ }^{15}$ E. Rasmussen, Naturwiss. 23, 69 (1935).

${ }_{16}$ P. Marmier and F. Boehm, Phys. Rev. 97, 103 (1955);

McClelland, Mark, and Goodman, Phys. Rev. 97, 1191 (1955); N. P. Heydenburg and G. M. Temmer, Phys. Rev. (to be published).

${ }^{17}$ For further details of this calculation see reference 5 .

18 This change is revealed especially in the quadrupole moments of $\mathrm{Eu}^{151}$ and $\mathrm{Eu}^{153}$ [see P. Brix, Z. Physik 132, 579 (1952)], in the excitation energy of the first excited states of the even-even nuclei [see, e. g., G. Scharff-Goldhaber, Phys. Rev. 90, 587 (1953)] and in the atomic isotope shift data [see P. Brix and H. Kopferman, Z. Physik 126, 344 (1944)].

\section{Mean Lifetime of Positive $K$ Mesons*}

E. L. Iloff, W. W. Chupp, Gerson Goldhaber, S. Goldhaber, AND J. E. LANNUTTr, Radiation Laboratory and Department of Physics, University of California, Berkeley, California

AND

A. Pevsner and D. Ritson, Massachusetts Institute of Technology, Cambridge, Massachusetts

(Received July 11, 1955)

$\mathbf{M}$ EAN lifetimes for heavy mesons from cosmic rays have been reported by various groups using cloud chambers and Čerenkov counters. ${ }^{1,2}$ We have carried out a measurement of the mean lifetime of artificially produced $\mathrm{K}^{+}$mesons by making use of their decay in flight in nuclear emulsion. 